\title{
On Uryson Operators with Partial Integrals in Lebesgue Spaces with Mixed Norm
}

\author{
C.-J. Chen and A. S. Kalitvin
}

\begin{abstract}
In this paper we consider some class of partial Uryson integral operators in spaces with mixed norm. We give some conditions for action, boundedness and continuity of those operators.
\end{abstract}

Keywords: Nonlinear Uryson operators, partial integral operators, acting conditions, boundedness conditions, continuity conditions

AMS subject classification: Primary 47 H 30, secondary 45 P 05, 45 G 10

\section{Introduction}

Let $T \subset \mathbb{R}^{n}$ and $S \subset \mathbb{R}^{m}$ be two compact sets with Lebesgue measure, $D=T \times S$, $a_{i}: D \times \mathbb{R} \rightarrow \mathbb{R}(i=1,2,3)$ given Carathéodory functions, and

$$
A=A_{1}+A_{2}+A_{3},
$$

where

$$
\begin{aligned}
& \left(A_{1} x\right)(t, s)=\int_{T} a_{1}(t, s, x(\tau, s)) d \tau \\
& \left(A_{2} x\right)(t, s)=\int_{S} a_{2}(t, s, x(t, \sigma)) d \sigma \\
& \left(A_{3} x\right)(t, s)=\iint_{D} a_{3}(t, s, x(\tau, \sigma)) d \tau d \sigma .
\end{aligned}
$$

The operators $A, A_{1}$, and $A_{2}$ are so called partial Uryson integral operators, which have been studied in $C(D)$, in spaces with mixed quasinorm $L^{\alpha}\left[L^{\beta}\right]$, and in quasi-Banach ideal spaces (see $[1,4,8]$, respectively). The properties of partial Uryson integral operators essentially differ from those of ordinary Uryson integral operators. For example, the operator $A_{1}$ with kernel $a_{1}(t, s, u) \equiv u$ is not completely continuous in $L^{P}(D)$, but the operator $A_{3}$ is completely continuous for $a_{3}(t, s, u) \equiv u$.

A. S. Kalitvin: Pedag. Inst., Dept. Math., ul. Lenina, R - 398020 Lipetsk, Russia Chen Chur-jen: University of Würzburg, Dept. Math., Am Hubland, D - 97074 Würzburg Financial support by the DAAD Bonn (Kz. A/95/08858) is gratefully acknowledged.

ISSN 0232-2064 / \$2.50 (C) Heldermann Verlag Berlin 
We remark that linear and nonlinear operators with partial integrals have applications in problems of continuum mechanics, of the theory of transfer, of differential and integro-differential equations and other questions.

In this paper the operator $A$ is studied in Lebesgue spaces with mixed norm

$$
L^{q}(T)\left[L^{p}(S)\right] \quad(1 \leq p, q \leq \infty)
$$

More general classes of partial Uryson integral operators in spaces of summable functions have been studied in [3]. Action, boundedness, and continuity criteria of the operator $A_{3}$ in Lebesgue spaces have been obtained by Ojnarov [6].

\section{Action, boundedness and continuity}

Let $M(D)$ be the space of all real measurable almost everywhere finite functions on $D$. We denote by $L^{q}(T)\left[L^{p}(S)\right](1 \leq p, q \leq \infty)$ the set of functions $x \in M(D)$ for which the norm

$$
\|x\|_{L^{q}(T)\left[L^{P}(S)\right]}=\|t \mapsto\| x(t, \cdot)\left\|_{L^{P}(S)}\right\|_{L^{\Phi}(T)}
$$

is defined and finite [2]. These spaces are Banach spaces. Of course, in case $p=q$ we have $L^{p}(T)\left[L^{p}(S)\right] \cong L^{p}(T \times S)$. Let $X\left(x_{0}, r\right)$ denote the closed ball of radius $r$ with center $x_{0}$ in the space $X$.

The operators $A_{1}, A_{2}$, and $A_{3}$ are defined on functions $x \in M(D)$ for which the functions

$$
\begin{aligned}
(t, s, \tau) & \longmapsto a_{1}(t, s, x(\tau, s)) \\
(t, s, \sigma) & \longmapsto a_{2}(t, s, x(t, \sigma)) \\
(t, s, \tau, \sigma) & \longmapsto a_{3}(t, s, x(\tau, \sigma))
\end{aligned}
$$

are summable in the variables $\tau, \sigma$, and $(\tau, \sigma)$, respectively, for almost all $(t, s) \in D$. Let $D(A)$ be the domain of definition of the operator $A=A_{1}+A_{2}+A_{3}$. If $a_{1}(t, s, 0)=$ $a_{2}(t, s, 0)=a_{3}(t, s, 0)=0$, and $x_{1}, \ldots, x_{n} \in D(A)$ are functions with disjoint supports, then $x_{1}+\ldots+x_{n} \in D(A)$ and the operator $A$ is partially additive, i.e.,

$$
A\left(x_{1}+\ldots+x_{n}\right)=A x_{1}+\ldots+A x_{n} .
$$

In general, the operator $x \mapsto A\left(x+x_{0}\right)-A x_{0}$ is partially additive for fixed $x_{0} \in D(A)$.

By the partial additivity of $A$ we have the following statement.

Theorem 1. Let $X=L^{q}(T)\left[L^{p}(S)\right] \quad(1 \leq p, q<\infty)$. Suppose that the operator $A$ acts from $X\left(x_{0}, r\right)$ into $Y=L^{\beta}(T)\left[L^{\alpha}(S)\right](1 \leq \alpha, \beta \leq \infty)$. Then $A$ acts from $X$ into $Y$ and is bounded (i.e., $A$ is bounded on any bounded set). Moreover, $A$ is continuous on $X$ if $A$ is continuous on $X\left(x_{0}, r\right)$.

By Theorem 1 the boundedness of the operator $A$ follows direct from its action.

The next theorem concerning acting conditions (both sufficient and necessary) for the operator $A_{3}$ may be obtained following the idea of [6]. 
Theorem 2. The operator $A_{3}$ acts from $X=L^{q}(T)\left[L^{p}(S)\right](1 \leq p, q<\infty)$ into $Y=L^{\beta}(T)\left[L^{\alpha}(S)\right] \quad(1 \leq \alpha, \beta \leq \infty)$ if and only if, for any $u \in \mathbb{R}$,

$$
\left\|a_{3}(\cdot, \cdot, u)\right\|_{Y} \leq a|u|^{\min \{p, q\}}+b
$$

where $a$ and $b$ are non-negative constants.

Proof. Without loss of generality, we assume that mes $T=\operatorname{mes} S=1$. Suppose that condition (4) holds. Then for any $x \in X$ the Hölder and Minkowski inequalities imply that

$$
\begin{aligned}
\left\|A_{3} x\right\|_{Y} & =\left\|\iint_{D} a_{3}(\cdot, \cdot, x(\tau, \sigma)) d \tau d \sigma\right\|_{Y} \\
& \leq \iint_{D}\left\|a_{3}(\cdot, \cdot, x(\tau, \sigma))\right\|_{Y} d \tau d \sigma \\
& \leq \iint_{D}\left(a|x(\tau, \sigma)|^{\min \{p, q\}}+b\right) d \tau d \sigma \\
& \leq a\|x\|_{X}^{\min \{p, q\}}+b .
\end{aligned}
$$

Hence, $A_{3}$ acts from $X$ into $Y$.

Conversely, suppose that the operator $A_{3}$ acts from $X$ into $Y$. Then, by Theorem 1 , there exists a number $b>0$ such that $\left\|A_{3} x\right\|_{Y} \leq b$ if $\|x\|_{X} \leq 1$. Let $u \in \mathbb{R}$ and $x_{u} \equiv u \in X$. If $|u| \leq 1$, it is clear that

$$
\left\|a_{3}(\cdot, \cdot, u)\right\|_{Y}=\left\|A_{3} x_{u}\right\|_{\gamma} \leq b \leq b\left(|u|^{\min \{p, q\}}+1\right) .
$$

If $|u|>1$, we define a function $\bar{x}_{u}$ on $D$ by

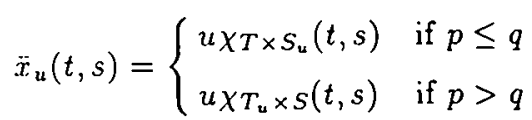

where $S_{u}$ is a measurable subset of $S$ with mes $S_{u}=|u|^{-p}$ and $T_{u}$ is a measurable subset of $T$ with mes $T_{u}=|u|^{-q}$. Here, $\chi_{T \times S_{u}}$ and $\chi_{T_{u} \times S}$ denote the characteristic functions of $T \times S_{u}$ and $T_{u} \times S$, respectively. Then $\left\|\bar{x}_{u}\right\|_{x}=1$ and

$$
\left\||u|^{-\min \{p, q\}} a_{3}(\cdot, \cdot, u)+\left(1-|u|^{-\min \{p, q\}}\right) a_{3}(\cdot, \cdot, 0)\right\|_{Y} \cdot=\left\|A_{3} \bar{x}_{u}\right\|_{Y} \leq b .
$$

Hence,

$$
\left\|a_{3}(\cdot, \cdot, u)\right\|_{Y} \leq b|u|^{\min \{p, q\}}+|u|^{\min \{p, q\}}\left\|a_{3}(\cdot, \cdot, 0)\right\| Y \leq 2 b|u|^{\min \{p, q\}} .
$$

From (5) and (6) it follows that condition (4) holds

Some acting conditions for the operators $A_{1}$ and $A_{2}$ in spaces of summable functions have been given in [3]. We will give simple acting conditions (only sufficient) in the next lemina. 
Lemma. Let $1 \leq p, q, \alpha, \beta<\infty, X=L^{q}(T)\left[L^{p}(S)\right]$, and $Y=L^{\beta}(T)\left[L^{\alpha}(S)\right]$. The operators $A_{1}$ and $A_{2}$ act from $X$ into $Y$ if the kernels $a_{1}$ and $a_{2}$ satisfy growth conditions of the form

$$
\left|a_{i}(t, s, u)\right| \leq c_{i}|u|^{\min \{p, q\} / \rho_{i}(\alpha, \beta)}+b_{i}(t, s) \quad(i=1,2)
$$

for some $b_{1}, b_{2} \in Y$ and $c_{1}, c_{2} \geq 0$, where $\rho_{1}(\alpha, \beta)=\alpha$ and $\rho_{2}(\alpha, \beta)=\beta$. Moreover, in this case $A_{1}$ and $A_{2}$ are bounded and continuous.

Proof. It is easy to show the first statement by the Hölder and Minkowski inequalities. The continuity of $A_{1}$ and $A_{2}$ follows from the principle of majorants [8]

We note that the growth condition (7) is not necessary for the action of $A_{1}$ (resp. $A_{2}$ ). Moreover, there exists $A_{1}$ acting from $X$ into $Y$ (whence $A_{1}$ is even bounded), which is not continuous. In particular, the corresponding kernel $a_{1}$ does not satisfy the growth condition (7) (by the previous lemma).

The following example is essentially due to P. P. Zabrejko [5].

Example. Let $D=[0,1] \times[0,1], X=L^{q}(T)\left[L^{p}(S)\right]$, and $Y=L^{\beta}(D)(1 \leq p, q, \beta<$ $\infty$ ). Let $z_{n}(t, s)=z_{n}(t) \geq 0$ have disjoint support, and $\left\|z_{n}\right\|_{Y}=1$. Define the kernel $a_{1}$ on $D \times \mathbb{R}$ by

$$
a_{1}(t, s, u)= \begin{cases}\left(2^{n}|u|-1\right) z_{n-1}(t)+\left(2-2^{n}|u|\right) z_{n}(t) & \text { if } 2^{-n} \leq|u|<2^{1-n} \\ 0 & \text { if } u=0 \text { or }|u| \geq 1\end{cases}
$$

Then the kernel $a_{1}$ is a non-negative Caratheodory function, and the operator $A_{1}$ acts from $X$ into $Y$ and is bounded (it even has bounded range): Indeed, by Minkowski's inequality we have for any measurable $x$

$$
\begin{aligned}
\left\|A_{1} x\right\|_{Y}^{\beta} & =\int_{0}^{1}\left\|\int_{0}^{1} a_{1}(\cdot, s, x(\tau, s)) d \tau\right\|_{L^{\beta}}^{\beta} d s \\
& \leq \int_{0}^{1}\left(\int_{0}^{1}\left\|a_{1}(\cdot, s, x(\tau, s))\right\|_{L^{\beta}} d \tau\right)^{\beta} d s \\
& \leq \int_{0}^{1}\left(\int_{0}^{1} 1 d \tau\right)^{\beta} d s \\
& \leq 1 .
\end{aligned}
$$

However, $A_{1}$ is not continuous, since it maps the convergent sequence $\left(x_{n}\right)=\left(2^{-n}\right)$ into the non-compact sequence $\left(A_{1} x_{n}\right)=\left(z_{n}\right)$.

The kernel $a_{1}$ not only fails to satisfy the growth condition (7). Even more, $a_{1}$ does not satisfy

$$
\left|a_{1}(t, s, u)\right| \leq c|u|^{\gamma}+b(t, s)
$$

for fixed $c, \gamma>0$ and $b \in Y$. Indeed, for $u_{n}=2^{-n},(8)$ would imply $z_{n}(t, s)=$ $a_{1}\left(t, s, u_{n}\right) \leq c+b(t, s)$, whence $d(t, s)=b(t, s)+c$ satisfies $d \geq z_{n}$ for all $n$, which obviously is not possible, since $d \in Y$. 
The continuity of the operator $A_{3}$ does not follow from its action and boundedness as is shown by the previous example (consider $a_{3}=a_{1}$ ).

To discuss continuity conditions for the operator $A_{3}$, we apply the following theorem. Recall that a set $G \subset X$ is absolutely bounded if $\sup \left\{\left\|\chi_{\Omega} x\right\|_{X}: x \in G\right\} \rightarrow 0$ as $\operatorname{mes} \Omega \rightarrow 0$.

Theorem 3. Let $1 \leq p, q, \alpha, \beta<\infty, X=L^{q}(T)\left[L^{p}(S)\right]$ and $Y=L^{\beta}(T)\left[L^{\alpha}(S]\right)$. Suppose that, for each function $x \in X$,

$$
\left\|\iint_{D}\left|a_{3}(\cdot, \cdot ; x(\tau, \sigma))\right| d \tau d \sigma\right\|_{Y}<\infty .
$$

Then the operator $A_{3}$ acts from $X$ into $Y$. Moreover, for each absolutely bounded set $G \subset X$ and for each $\varepsilon>0$ there exists a number $\delta>0$ such that the inequality

$$
\sup _{x \in G}\left\|\iint_{D_{1}}\left|a_{3}(\cdot, \cdot, x(\tau, \sigma))\right| d \tau d \sigma\right\|_{Y}<\varepsilon
$$

holds whenever $D_{1} \subset D$ satisfies $\operatorname{mes} D_{1}<\delta$.

Proof. It is analogous to that of [5: Theorem 18.4]

Theorem 4 (see [7]). Let $1 \leq p, q, \alpha, \beta<\infty$. The operator $A_{3}$ acts from $X=$ $L^{q}(T)\left[L^{p}(S)\right]$ into $Y=L^{\beta}(T)\left[L^{\alpha}(S)\right]$ and is continuous if and only if condition (4) holds and

$$
\lim _{u \rightarrow u_{0}}\left\|a_{3}(\cdot, \cdot, u)-a_{3}\left(\cdot, \cdot, u_{0}\right)\right\|_{Y}=0
$$

for any $u_{0} \in \mathbb{R}$.

Proof. Without loss of generality, assume $a_{3}(t, s, 0)=0$ and $\operatorname{mes} T=\operatorname{mes} S=1$. Suppose that the operator $A_{3}$ acts from $X$ into $Y$ and is continuous. Then condition (4) holds by Theorem 2. Putting $x \equiv u$ and $x_{0} \equiv u_{0}$, we have $A_{3} x=a_{3}(\cdot, \cdot, u)$ and $A_{3} x_{0}=a_{3}\left(\cdot, \cdot, u_{0}\right)$. Thus the continuity of $A_{3}$ implies (9).

Conversely, suppose that conditions (4) and (9) hold. Then the operator $A_{3}$ acts from $X$ into $Y$ by Theorem 2. Assume that $A_{3}$ is not continuous. This means that there exist a sequence $\left(x_{n}\right)$ converging to a function $x_{0}$ in $X$ and a number $\varepsilon_{0}>0$ such that

$$
\left\|A_{3} x_{n}-A_{3} x_{0}\right\|_{Y} \geq \varepsilon_{0} \quad(n \in \mathbb{N}) .
$$

Since $x_{n} \rightarrow x_{0}$ in $X$, the set $\left\{x_{0}, x_{1}, x_{2}, \ldots\right\}$ is absolutely bounded. Hence, by Theorem 3 there is a number $\delta>0$ such that the inequalities

$$
\left\|A_{3}\left(\chi_{F} x_{n}\right)\right\|_{Y}<\frac{\varepsilon_{0}}{3} \quad(n \geq 0)
$$

hold whenever $F \subset D$ satisfies mes $F<\delta$. Let $c=\sup _{n \geq 0}\left\|x_{n}\right\|_{X}, N=c\left(\frac{\delta}{3}\right)^{-1 / \min \{p, q\}}$, and $D_{n}^{N}=\left\{(t, s):\left|x_{n}(t, s)\right| \geq N\right\} \quad(n \geq 0)$. Then $\operatorname{mes} D_{n}^{N} \leq \frac{\delta}{3}(n \geq 0)$. Since $x_{n} \rightarrow x_{0}$ in $X$, we can find a subsequence $\left(x_{n_{k}}\right)$ which converges almost everywhere to $x_{0}$. Moreover, by Egorov's theoren, there exists a measurable set $D_{\delta} \subset D$ such that 
$\operatorname{mes}\left(D-D_{\delta}\right)<\frac{\delta}{3}$ and $\left(x_{n_{k}}\right)$ converges to $x_{0}$ uniformly on $D_{\delta}$. Let $F_{k}^{\delta}=D_{\delta}-\left(D_{n_{k}}^{N} \cup D_{0}^{N}\right)$ and $\tilde{F}_{k}^{\delta}=D-F_{k}^{\delta}(k \geq 1)$. Then mes $\tilde{F}_{k}^{\delta}<\delta$ for any $k \geq 1$. Now, we estimate

$$
\left\|A_{3} x_{n_{k}}-A_{3} x_{0}\right\|_{Y} \leq\left\|A_{3} \chi_{F_{k}^{6}} x_{n_{k}}-A_{3} \chi_{F_{k}^{6}} x_{0}\right\|_{Y}+\left\|A_{3} \chi_{\bar{F}_{k}^{\delta}} x_{n_{k}}\right\|_{Y}+\left\|A_{3} \chi_{\bar{F}_{k}^{\delta}} x_{0}\right\|_{Y} .
$$

By condition (9) there is a $\delta_{0}=\delta_{0}\left(N, \varepsilon_{0}\right)>0$ such that

$$
\left\|a_{3}(\cdot, \cdot, u)-a_{3}\left(\cdot, \cdot, u_{0}\right)\right\|_{Y}<\frac{\varepsilon_{0}}{3}
$$

whenever $|u|<N,\left|u_{0}\right|<N$, and $\left|u-u_{0}\right|<\delta_{0}$. Since $\left(x_{n_{k}}\right)$ converges to $x_{0}$ uniformly on $D_{\delta}$, there exists an integer $m=m\left(\delta_{0}\right)$ such that $\left|x_{n_{m}}(t, s)-x_{0}(t, s)\right|<\delta_{0}$ for all $(t, s) \in D_{\delta}$. Combining incqualities (11) - (13) we get $\left\|A_{3} x_{n_{m}}-A_{3} x_{0}\right\|_{Y}<\varepsilon_{0}$, which is contradictory to (10). Thus the operator $A_{3}$ is continuous

\section{References}

[1] Appell, J., De Pascale, E., and P. P. Zabreiko: On the application of the NewtonKantorovich method to nonlinear integral equations of Uryson type. Numer. Funct. Anal. Optim. 12 (1991), 271 - 283.

[2] Benedek, A. and R. Panzone: The spaces $L^{P}$, with mixed norm. Duke Math. J. 28 (1961), $301-324$.

[3] Chen, C.-J. and A. S. Kalitvin: Nonlinear operators with partial integrals in spaces of summable functions (submitted).

[4] Kalitvin, A. S.: Nonlinear operators with partial integrals. Proc. Sec. World Congr. Nonlin. Anal. (Athens, Greece) (to appear).

[5] Krasnoselskiǐ, M. A., Zabreǐko, P. P., Pustylnik, E. I. and P. E. Sobolevskiǐ: Integral operators in Spaces of Summable Functions. Leyden: Noordhoff 1976.

[6] Oinarov, R.: On the properties of a Uryson operator (in Russian). Dokl. Akad. Nauk SSSR 256 (1981), 281 - 284.

[7] Otelbaev, M. and G. A. Suvorchenkova: A necessary and sufficient condition for boundedness and continuity of a certain class of Uryson operators (in Russian). Sibirsk. Mat. Zh. 20 (1979), $428-431$.

[8] Povolotskiǐ, A. I. and A. S. Kalitvin: Nonlinear Partial Integral Operators (in Russian). St. Petersburg: Izd. Ross. Gos. Pedag. Univ. 1991. 